\title{
A comparison of CellCollector with Cellsearch in patients with neuroendocrine tumours
}

\section{Dear Editor,}

Circulating tumour cells (CTCs) have been hypothesised to be mediators of metastases (Fidler et al. 2003) but with numbers as low as $1 / 10^{7}$ white cells (Alunni-Fabbroni \& Sandri 2010), their utility as biomarkers has been limited by low rates of detection and isolation. CTCs have been identified in patients with metastatic neuroendocrine tumours (NETs) using the FDA-cleared CellSearch (Janssen Diagnostics) technology, a semi-automated platform that uses immunomagnetic enrichment of CTCs based on expression of epithelial cell adhesion molecule (EpCAM) (Khan et al. 2011). Using this platform, CTCs were found in $36 \%$ of patients with pancreatic NETs and $53 \%$ of those with midgut NETs. The presence of CTCs is associated with a worse overall survival, and early changes in CTC number after treatment in NET patients are also prognostic (Khan et al. 2013, 2016). CTCs may also be considered as 'liquid biopsies', offering the opportunity to interrogate the molecular characteristics of the tumour. For such an approach to be broadly applicable, alternative technologies are required to increase number of CTCs isolated and the proportion of patients in whom they can be detected.

The CellCollector (GILUPI GmbH, Potsdam, Germany) is a novel medical device consisting of a $160 \mathrm{~mm}$ sterile steel wire of which the terminal $20 \mathrm{~mm}$ is coated with anti-EpCAM antibodies covalently coupled to a gold and hydrogel layer. The CellCollector is inserted into a peripheral vein enabling the circulating blood volume to be sampled. The wire is stained with fluorescently labelled antibodies and examined microscopically to identify CTCs. The clinical application of this device has been previously reported in patients with breast and lung cancer (Saucedo-Zeni et al. 2012).

In this study, we sought to compare the performance of theCellCollector and CellSearchin patientswith metastatic NETs. Thirty-four patients provided written informed consent and were recruited into the study (Table 1).
The protocol was approved by the central ethical review board (IRAS Project ID 105772). The CellCollector was inserted into the cubital vein via a $20 \mathrm{G}$ cannula and left in situ for $30 \mathrm{~min}$ after which it was removed, washed in phosphate buffered saline (PBS) and fixed in acetone. The cells were permeabilised (Triton X-100 in PBS, 0.1\% concentration) at room temperature, washed in PBS and incubated with blocking buffer (bovine serum albumin (BSA)/PBS, 3\% concentration). Immunostaining was performed with a solution containing FITCconjugated antibodies against EpCAM (1:50; HEA125, Acris Antibodies, Herford, Germany), cytokeratin 19 conjugated with Alexa488 (1:50, A53-B/A2, Life Technologies), pan-cytokeratin-Alexa488 (1:50, C11, eBioscience, San Diego, CA, USA) and cytokeratin 7-FITC (1:50, LP5K Milipore). An Alexa-Fluor 647-conjugated anti-CD45 rabbit polyclonal antibody was added as negative marker to exclude white blood cells (1:25, MEM-28Exbio, Czech Republic). Finally, the wire was incubated in the nuclear stain, Hoesch 33342 (Sigma), (concentration $1 \mu \mathrm{g} / \mathrm{mL}$ ). The wire was examined in a bespoke holder allowing inspection in four planes using an Axio Imager microscope with digital camera and AxioVision software.

CTCs were defined according to the following criteria: (1) intact cellular morphology, (2) cell diameter more than $4 \mu \mathrm{m}$, (3) positive for cytokeratin and nuclear stain, but negative for CD45 and (4) nuclear stain distinct from the cytokeratin or EpCAM staining. Examples of positively identified CTCs are shown in Fig. 1. The number of CTCs was enumerated by two independent operators who were blind to the patient's clinical information. Where there was disagreement between the two operators, a third operator arbitrated. A $7.5 \mathrm{~mL}$ peripheral blood sample was collected concurrently into a CellSave tube and analysed within $72 \mathrm{~h}$ by CellSearch as described previously (Khan et al. 2011).

Published by Bioscientifica Ltd 
Table 1 Clinicopathological details of study cohort.

\begin{tabular}{|c|c|c|c|}
\hline Primary & Midgut $(n=18)$ & PNET $(n=14)$ & Other $(n=3)$ \\
\hline $\begin{array}{l}\text { Age: median } \\
\text { range }\end{array}$ & $\begin{array}{c}59 \\
(40-74)\end{array}$ & $\begin{array}{c}58.6 \\
(36-66)\end{array}$ & $\begin{array}{c}50 \\
(40-56)\end{array}$ \\
\hline Sex: Female & 5 & 6 & 3 \\
\hline Male & 13 & 8 & \\
\hline Median duration & 64.5 & 32 & 62 \\
\hline$<25 \%$ Liver disease & 8 & 7 & 1 \\
\hline$>25 \%$ Liver disease & 10 & 7 & 2 \\
\hline Primary resection & 11 & 2 & 2 \\
\hline Grade 1 & 15 & 2 & 0 \\
\hline Grade 2 & 3 & 10 & 2 \\
\hline Grade 3 & 0 & 2 & 1 \\
\hline \multicolumn{4}{|l|}{ Metastatic sites } \\
\hline Lymph node & 16 & 12 & 2 \\
\hline Bone & 4 & 3 & 2 \\
\hline Lung & 1 & 1 & 1 \\
\hline Peritoneal & 10 & 1 & 1 \\
\hline Brain & 0 & 0 & 0 \\
\hline Other & 2 & 1 & 0 \\
\hline \multicolumn{4}{|l|}{ Previous therapy } \\
\hline SST analogues & 13 & 5 & 2 \\
\hline Chemotherapy & 1 & 5 & 1 \\
\hline TAE & 1 & 0 & 0 \\
\hline RFA & 1 & 0 & 1 \\
\hline PRRT & 3 & 2 & 0 \\
\hline Sunitinib & 0 & 0 & 0 \\
\hline Everolimus & 0 & 0 & 0 \\
\hline Interferon & 1 & 0 & 0 \\
\hline
\end{tabular}

PRRT, peptide radiotargeted receptor therapy; RFA, radiofrequency ablation; SST, somatostatin; TAE, transarterial embolisation.

The interobserver variation for CellSearch has been previously reported (Allard et al. 2004), and here we demonstrated good correlation between observers enumerating CTCs using the CellCollector achieving Spearman's correlation of 0.92 (95\% CI: $0.85,0.96)$ $(P<0.0001)$ (Fig. 2A). The median number of CTCs enumerated with CellCollector was 6 (range 2-49), compared with a median of 0 (range 0-57) with CellSearch $(P<0.0001$ (Mann-Whitney $U$ test)). In 33 of 34 patients, there was $\geq 1$ CTC found compared with only 16 of 34 patients with CellSearch (Table 2). Therefore, CTCs were detected in greater numbers and a greater proportion of patients with the CellCollector (Fig. 2B). The CellCollector identified CTCs in all midgut NETs and 12 of 13 PNETS.

We explored the prognostic relevance of CTC count according to CellCollector. With a median follow-up period of 13 months, overall survival data were insufficiently mature so we examined progression-free survival (PFS) as a surrogate. Overall, 14 patients had progression by RECIST criteria, and applying a cutoff of 7 CTCs, there was a significant difference in PFS (Cox Hazard Ratio 3.4, $P<0.05)$. Using the same threshold in the Kaplan-Meier survival analyses (Fig. 2C), median PFS was 11 months for patients with $\geq 7$ CTCs but not reached for those with $<7$ (log-rank, $P<0.05)$.

Here, we have demonstrated for the first time that the CellCollector is able to detect CTCs in more NET patients and in greater numbers than CellSearch. However, the CellSearch has been extensively validated and remains a robust method for prognostication, whereas the CellCollector offers the potential to make molecular analysis of CTCs more widely applicable. Indeed, a recent study in lung cancer demonstrated both KRAS and EGFR mutations known to be present in the primary tumour, in CTCs derived from the CellCollector using chip-based digital PCR (Gorges et al. 2016). Other strategies to increase the volume of blood sampled for CTCs include the use of leukapheresis (Fischer et al. 2013). However, the leukapheresis product has a very high rate

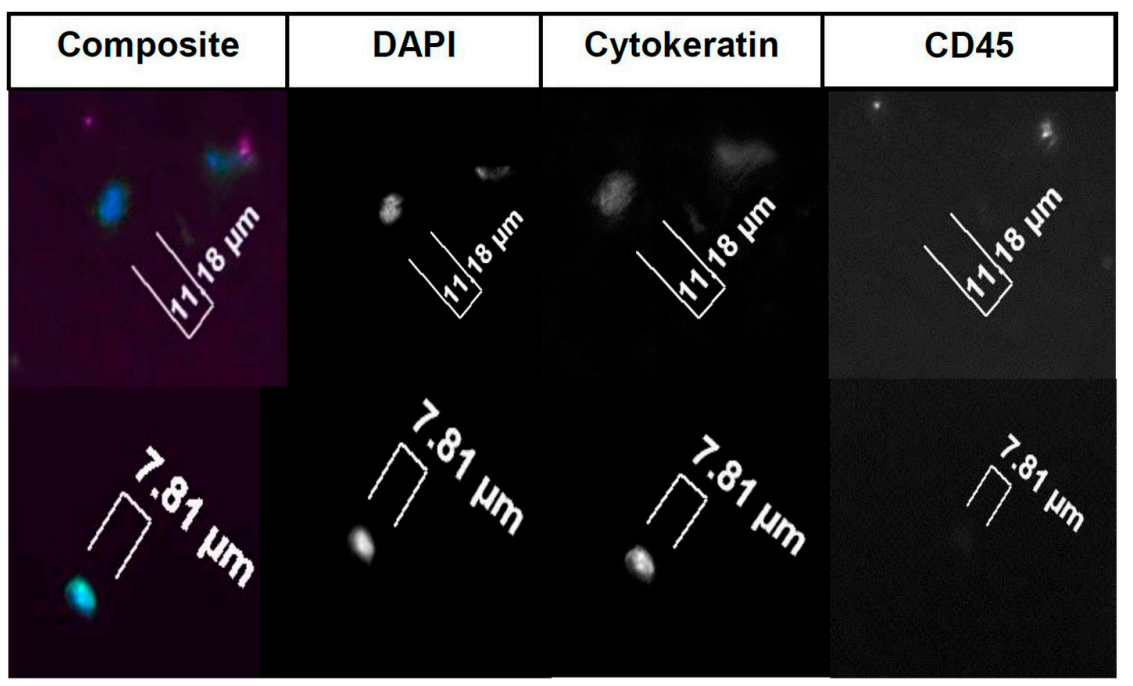

Figure 1

Examples of CTCs identified using immunofluorescent microscope, with signal for each channel demonstrated alongside composite image. http://erc.endocrinology-journals.org DOI: 10.1530/ERC-16-0201
() 2016 Society for Endocrinology Printed in Great Britain
Published by Bioscientifica Ltd 

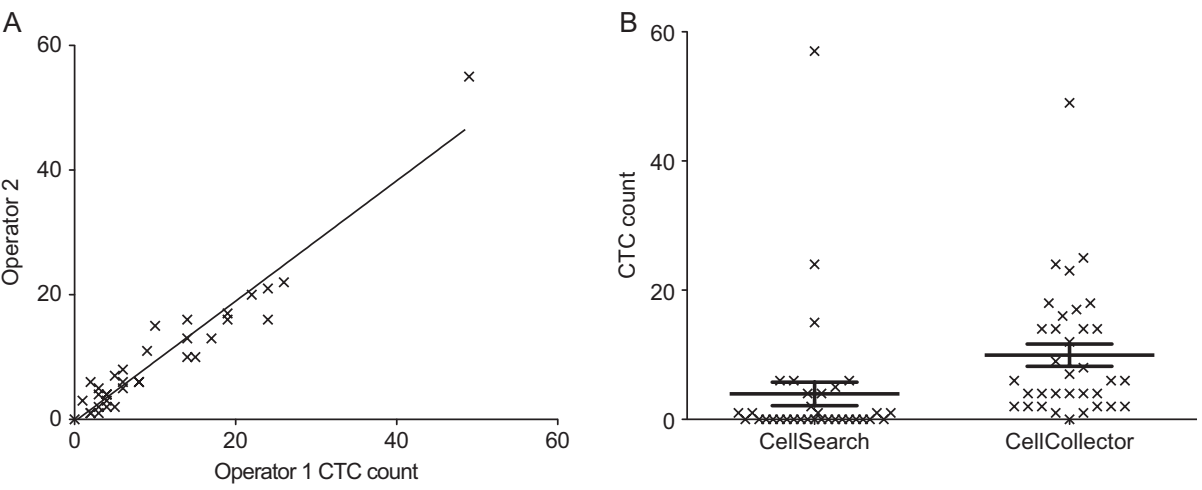

C

Figure 2

(A) Correlation between CTC identified by each operator for each wire enumerated. (B) Scattergram CTCs identified by CellCollector compared with CellSearch. (C) Kaplan-Meier survival for PFS when using 7 CTCs as threshold.

Table 2 Demonstrates CTC count from both CellCollector and CellSearch for all 34 patients who underwent successful enumeration with each isolation method.

\begin{tabular}{|c|c|c|c|c|c|c|c|}
\hline Pt No. & Age & Primary & Grade & CTC by CellSearch & CTC by CellCollector & $>25 \%$ Liver metastases & $\geq 3$ sites of metastases \\
\hline CD02 & 74 & Midgut & 1 & 4 & 14 & Yes & Yes \\
\hline CD03 & 59 & Midgut & 1 & 1 & 2 & Yes & Yes \\
\hline CD04 & 65 & PNET & 2 & 0 & 2 & No & Yes \\
\hline CD05 & 72 & PNET & 1 & 1 & 1 & Yes & Yes \\
\hline CD06 & 56 & Hindgut & 2 & 1 & 1 & Yes & Yes \\
\hline CD07 & 71 & Midgut & 1 & 6 & 2 & No & Yes \\
\hline CD08 & 64 & PNET & 2 & 0 & 4 & Yes & Yes \\
\hline CD09 & 59 & Midgut & 1 & 1 & 2 & No & No \\
\hline CD10 & 64 & PNET & 2 & 0 & 4 & Yes & No \\
\hline CD11 & 66 & PNET & 2 & 0 & 4 & Yes & No \\
\hline CD12 & 52 & PNET & 3 & 0 & 2 & No & No \\
\hline CD13 & 61 & Midgut & 1 & 1 & 4 & No & No \\
\hline CD14 & 65 & PNET & 3 & 6 & 9 & Yes & Yes \\
\hline CD15 & 50 & Bronchial & 2 & 0 & 2 & No & Yes \\
\hline CD16 & 54 & Midgut & 1 & 57 & 49 & Yes & No \\
\hline CD17 & 54 & PNET & 1 & 0 & 8 & No & No \\
\hline CD18 & 36 & PNET & 2 & 0 & 0 & No & No \\
\hline CD19 & 40 & Midgut & 2 & 0 & 4 & Yes & Yes \\
\hline CD20 & 67 & Midgut & 1 & 0 & 17 & Yes & No \\
\hline $\mathrm{CD} 21$ & 54 & Midgut & 1 & 0 & 6 & No & No \\
\hline CD22 & 66 & PNET & 2 & 0 & 24 & No & Yes \\
\hline CD23 & 57 & Midgut & 1 & 0 & 14 & Yes & No \\
\hline CD24 & 68 & Midgut & 1 & 0 & 14 & Yes & Yes \\
\hline CD25 & 54 & Midgut & 1 & 0 & 6 & No & Yes \\
\hline CD26 & 44 & Unknown & 3 & 24 & 25 & Yes & Yes \\
\hline $\mathrm{CD} 27$ & 65 & Midgut & 1 & 0 & 16 & Yes & No \\
\hline CD28 & 51 & PNET & 2 & 0 & 18 & Yes & Yes \\
\hline CD29 & 44 & Midgut & 2 & 0 & 4 & No & No \\
\hline CD30 & 66 & PNET & 2 & 5 & 14 & Yes & Yes \\
\hline CD31 & 70 & Midgut & 1 & 0 & 6 & No & Yes \\
\hline CD32 & 72 & Midgut & 1 & 4 & 18 & No & No \\
\hline CD33 & 50 & Midgut & 2 & 6 & 7 & Yes & Yes \\
\hline CD34 & 44 & Midgut & 1 & 15 & 23 & Yes & Yes \\
\hline CD35 & 69 & PNET & 2 & 2 & 12 & No & Yes \\
\hline
\end{tabular}


of contaminating leucocytes and requires downstream enrichment methods to isolate CTCs. Compared with CellCollector, leukapheresis is also more time-consuming, expensive and onerous for patients (Stoecklein et al. 2016).

The CellCollector, like CellSearch, is limited by the dependence on EpCAM as a selection marker for CTCs, and a biologically important component of EpCAMnegative CTCs will not be sampled by either technology. Marker agnostic devices based on size exclusion or biophysical properties rather than antigen expression, offer an alternative method of CTC isolation but remain limited by the small volume of blood that can be sampled.

In summary, the CellCollector appears to be a promising innovation that may help enhance our understanding of CTC biology and the mechanism of metastasis.

D Mandair ${ }^{1,2}$
C Vesely ${ }^{1}$
L Ensell1$^{1}$
H Lowe $^{1}$
V Spanswick ${ }^{1}$
J A Hartley ${ }^{1}$
M E Caplin ${ }^{2}$
T Meyer ${ }^{1,2}$
${ }^{1}$ UCL Cancer Institute, University College London,
London, UK
${ }^{2}$ Neuroendocrine Tumour Unit, Royal Free Hospital,
London, UK

(Correspondence should be addressed to T Meyer; email: t.meyer@ucl.ac.uk)

\section{Declaration of interest}

The authors declare that there is no conflict of interest that could be perceived as prejudicing the impartiality of the research reported.

\section{Funding}

TM is part funded by the NIHR UCL Hospitals Biomedical Research Centre and the work was supported by the NIHR UCLH Clinical Research Facility and the UCL Experimental Cancer Medicine Centre. DM is funded by Royal Free Charities and Quiet Cancer appeal.

\section{References}

Allard WJ, Matera J, Miller MC, Repollet M, Connelly MC, Rao C, Tibbe AG, Uhr JW \& Terstappen LW 2004 Tumor cells circulate in the peripheral blood of all major carcinomas but not in healthy subjects or patients with nonmalignant diseases. Clinical Cancer Research 10 6897-6904. (doi:10.1158/1078-0432. CCR-04-0378)

Alunni-Fabbroni M \& Sandri MT 2010 Circulating tumour cells in clinical practice: methods of detection and possible characterization. Methods 50 289-297. (doi:10.1016/j.ymeth.2010.01.027)

Fidler IJ 2003 The pathogenesis of cancer metastasis: the 'seed and soil' hypothesis revisited. Nature Reviews Cancer 3 453-458. (doi:10.1038/nrc1098)

Fischer JC, Niederacher D, Topp SA, Honisch E, Schumacher S, Schmitz N, Zacarias Fohrding L, Vay C, Hoffman I, Kaspowicz NS, et al. 2013 Diagnostic leukapheresis enables reliable detection of circulating tumor cells of nonmetastatic cancer patients. PNAS 110 16580-16585. (doi:10.1073/pnas.1313594110)

Gorges TM, Penkaila N, Schalk T, Joosse SA, Eiethdorf S, Tucholski J, Lucke K, Wikman H, Jackson S, Brychta N, et al. 2016 Enumeration and molecular characterization of tumour cells in lung cancer patients using a novel in vivo device for capturing circulating tumour cells. Clinical Cancer Research 22 2197-2206. (doi:10.1158/1078-0432.CCR-15-1416)

Khan MS, Tsigani T, Rashid M, Rabouhans JS, Yu D, Luong TV, Caplin ME \& Meyer T 2011 Circulating tumor cells and EpCAM expression in neuroendocrine tumors. Clinical Cancer Research 17 337-345. (doi:10.1158/1078-0432.CCR-10-1776)

Khan MS, Kirkwood A, Tsigani T, Garcia-Hernandez J, Hartley JA, Caplin ME \& Meyer T 2013 Circulating tumor cells as prognostic markers in neuroendocrinetumors. Journal of Clinical Oncology $\mathbf{3 1}$ 365-372. (doi:10.1200/JCO.2012.44.2905)

Khan MS, Kirkwood AA, Tsigani T, Lowe H, Goldstein R, Hartley JA, Caplin ME \& Meyer T 2016 Early changes in circulating tumor cells are associated with response and survival following treatment of metastatic neuroendocrine neoplasms. Clinical Cancer Research 22 79-85. (doi:10.1158/1078-0432.CCR-15-1008)

Saucedo-Zeni N, Mewes S, Niestroj R, Gasiorowski L, Murawa D, Nowaczyk P, Tomasi T, Weber E, Dworacki G, Morgenthaler NG, et al. 2012 A novel method for the in vivo isolation of circulating tumor cells from peripheral blood of cancer patients using a functionalized and structured medical wire. International Journal of Oncology 41 1241-1250. (doi:10.3892/ijo.2012.1557)

Stoecklein NH, Fischer JC, Niederacher D \& Terstappen LW 2016 Challenges for CTC-based liquid biopsies: low CTC frequency and diagnostic leukapheresis as a potential solution. Expert Review of Molecular Diagnostics 16 147-164. (doi:10.1586/14737159. 2016.1123095)

Received in final form 8 August 2016

Accepted 12 August 2016

Accepted Preprint published online 12 August 2016
C 2016 Society for Endocrinology Printed in Great Britain
Published by Bioscientifica Ltd 